\title{
Teorías subjetivas sobre disciplina parental en literatura para padres
}

\section{Subjective theories about parental discipline in parenting literature}

\author{
Pablo Castro Carrasco ${ }^{a}$, David Cuadra-Martínez ${ }^{\mathfrak{b}}$, Carolina Hu González ${ }^{\mathfrak{c}}$ \\ aPsicólogo, Doctor en Psicología. Departamento de Psicología, Facultad de Humanidades, Universidad de La Serena, Chile. \\ Universidad Católica del Maule, Chile. Fundación América por la Infancia \\ bPsicólogo, Magíster en Psicología mención Psicología Educacional, Universidad de Atacama. Chile \\ cMédico-Pediatra. Investigadora Independiente. Alemania
}

Recibido el 28 de junio de 2018; aceptado el 23 de octubre de 2018

\section{Resumen}

Introducción: La disciplina parental es un tema de frecuente solicitud de orientación de quienes consultan a profesionales de la salud. Se relaciona con los estilos de crianza y ha sido estudiada científicamente respecto a su efectividad y consecuencias para los hijos(as). Objetivo: Describir teorías subjetivas presentes en las recomendaciones que efectúan los médicos y psicólogos en libros para padres en español acerca de la disciplina parental. Material y Método: Se analizaron trece libros con consejos para padres escritos por profesionales de la salud en idioma español publicados entre los años 2000 y 2017. El análisis de datos utilizó procedimientos de la Grounded Theory adaptados para el estudio de teorías subjetivas, mediante codificación abierta y axial. Además se hizo codificación temática para describir los casos, identificando lemas y teorías subjetivas para cada caso, las que a su vez se agruparon en teorías subjetivas supraordenadas. Resultados: Los datos fueron agrupados en tres teorías subjetivas generales acerca de la disciplina familiar en los libros analizados. Estas teorías subjetivas difieren entre ellas en la importancia otorgada a la autoridad, al castigo y a lo necesario de la disciplina parental. Conclusión: Hay discrepancias sobre disciplina familiar entre los libros. Se presenta poca evidencia científica en las teorías subjetivas de los libros. Los resultados difieren de los análisis históricos realizados sobre estas publicaciones en otros contextos culturales y temporales. Se destaca la importancia de desarrollar guías prácticas sobre disciplina parental basadas en la evidencia, para orientar a los padres por parte de los profesionales de la salud.
Palabras clave: Crianza de los hijos; Padres; Publicaciones; Investigación cualitativa; Educación del paciente; Relaciones padres-hijo

\begin{abstract}
Introduction: Parental discipline is a topic frequently asked for advice by those who consult health professionals. It is related to parenting styles and has been scientifically studied regarding its effectiveness and impact on children. Objective: To describe the subjective theories about parental discipline present in recommendations made by doctors and psychologists in books for parents in Spanish. Material and Method: Thirteen books with advice for parents, written in Spanish by healthcare profes-

Keywords: Parenting; Parents; Publications; Qualitative research; Patient education; parent-child relationships
\end{abstract}


sionals published between 2000 and 2017 were analyzed. Data analysis was performed using Grounded Theory procedures adapted for the study of subjective theories, through open and axial coding. In addition, thematic codification was made to describe the cases, identifying subjective mottos and subjective theories for each case, which at the same time were grouped into superordinate subjective theories. Results: The data were grouped into three general subjective theories about parental discipline in the books analyzed. These subjective theories differ from one another in the importance attributed to authority, punishment, and the necessity of parental discipline. Conclusion: There are differences in parental discipline among the books. There is little scientific evidence on the subjective theories of the books. Results differ from historical analyses of these publications in other cultural and temporal contexts. The importance of developing practical guidelines on parental discipline based on evidence to guide parents by health professionals is highlighted.

\section{Introducción}

La disciplina parental (DP) es un tema de frecuente preocupación en la crianza de los niños(as) y adolescentes, en el que confluyen valores personales, la cultura, la propia experiencia y, cada vez con más importancia, la evidencia científica. En variadas ocasiones los profesionales de la salud son consultados por los padres sobre la temática y éstos los asesoran desde el conocimiento científico y profesional que poseen, pero además desde las llamadas "teorías subjetivas" (TS) que sustentan y que corresponden a hipótesis o explicaciones personales no objetivas o científicas ${ }^{1}$.

Las TS y su relación con las prácticas, constituyen un área de estudio en profesionales de la salud mostrando que las TS influyen en su ejercicio profesional ${ }^{2,3}$. En otros profesionales, por ejemplo, en profesores es un enfoque largamente estudiado para la relación entre sus prácticas y las teorías científicas 4 .

En áreas relacionadas con la vida personal del profesional, por ejemplo, la crianza del niño(a), es probable que las TS tengan una influencia importante, a veces mayor, a la de las teorías científicas. Las TS no son determinadas por las experiencias personales de una persona, pero sí es indudable que en áreas relativas a lo socio moral la influencia puede llegar a ser importante. Lo anterior puede generar la entrega de información diferente según el profesional al que acuden los padres, escuchen o lean, generando, por ejemplo, frustración en ellos ${ }^{5}$.

La DP es el conjunto de actos y reglas que los padres usan para promover en los niños(as) valores y comportamientos, mediante distintos estilos como el democrático, autoritario, autoritativo y permisivo. Tiene un efecto significativo en el bienestar de los niños(as) porque guía el desarrollo moral, emocional y físico ${ }^{6}$.

Los consejos para padres dados por profesionales de la salud a través de diferentes medios, han sido estudiados ${ }^{7}$. El medio más clásico y estudiado son los libros de crianza escritos para padres ${ }^{8}$. Estudios muestran que el contenido de las recomendaciones está influido no solo por el conocimiento en el área del profesional, sino que influyen también el contexto cultural, las creencias personales y las tendencias de moda ${ }^{9,10,11}$.

La evidencia científica acerca de la DP señala que: (a) una combinación entre la crianza con autoridad, el amor y límites definidos influiría en un mejor autocontrol, autosuficiencia, iniciativa y desarrollo cognitivo ${ }^{12}$; (b) la asociación de la estrategia verbal, el tiempo fuera (time out) y las estrategias físicas no abusivas, reducen el comportamiento no cooperativo y agresivo del niño(a $)^{13}, y(c)$ el uso de una disciplina excesivamente punitiva o exageradamente permisiva, conllevaría a un deterioro de la salud del niño(a ${ }^{12}$ y a problemas en su comportamiento $^{14}$

$\mathrm{Al}$ respecto, hay concepciones que centran esta disciplina en la dureza en el trato. Sobre el castigo físico hay evidencia disponible sobre sus consecuencias negativas para el desarrollo del niño(a $)^{15,16}$ al igual que el castigo psicológico ${ }^{17}$.

No obstante, a pesar de la relevancia de esta temática para el desarrollo del niño(a), Hispanoamérica y España carecen de estudios concluyentes que ofrezcan una perspectiva acerca de cómo se está apoyando a los padres en esta tarea. Una forma de contribuir a lo anterior, es aproximándonos a las TS presentes en los consejos dados por profesionales de la salud, a través de libros para padres.

De acuerdo todo lo anterior, las siguientes preguntas generativas ${ }^{18}$ orientan la presente investigación:

1. ¿Existirá una TS general compartida sobre la DP, en los libros estudiados?

2. ¿Se observan en los libros analizados tendencias similares a las encontradas en estudios de este tipo de libros, en contextos diferentes al latinoamericano?

3. ¿Existirá evidencia científica para las TS sobre DP en los libros analizados?

El objetivo general de este estudio es comprender las TS presentes en libros para padres, en idioma español y de autores(as) profesionales de la salud, acerca de 
la DP. Específicamente interesa, 1) describir e interpretar las TS presentes en los libros; 2) comparar entre los casos las TS reconstruidas y 3 ) evaluar la presencia de evidencia científica que sustente las TS contendidas en las recomendaciones dadas en los libros.

\section{Material y Método}

Estudio transversal y descriptivo. La investigación se enmarca dentro del enfoque cualitativo, por lo tanto, no procede incluir hipótesis previas a la recogida de datos. Sin embargo enunciamos previamente preguntas generativas en su lugar.

Para la recolección de información se utilizó la revisión de 35 libros para padres, escritos en español desde el año 1994, en el contexto de un trabajo previo realizado con anterioridad por el primer autor del presente estudio ${ }^{19}$. En dicho estudio se encontró que el $66 \%$ de ellos $(n=23)$ incluía como tema la disciplina familiar, para esto se utilizaron los siguientes términos de búsqueda: "disciplina”, límites y "castigo(s)" entre marzo y diciembre de 2016.

Como arranque muestral se consideraron para el presente estudio los 23 libros identificados en el estudio anterior que incluían la temática de disciplina, de ellos se seleccionaron, para el análisis del presente trabajo, los que cumplieran con los siguientes criterios de inclusión: que la edición del libro analizada haya sido publicada desde el año 2000 en adelante, que los autores sean profesionales de la salud y que no hubiese en los 23 más de 1 libro por autor(a). Siguiendo estos criterios fueron excluidos 10 libros. Se excluyeron 2 libros de autores(as) que tenían más de un libro publicado en el periodo para no generar una "sobre representación" de sus TS en el análisis. También se excluyeron 8 libros publicados con anterioridad al año 2000. Posterior a esto se reemplazó 1 libro de los 13 incluidos, por su última edición disponible a la venta (2017).

La muestra quedó conformada por 13 libros. Todos disponibles para comprar en o desde Chile (on line) en el periodo de búsqueda. Estos se enumeran en tabla 1.

El análisis de datos se basó en procedimientos de la Grounded Theory ${ }^{20}$ y el procedimiento de descripciones breves de caso de la codificación temática ${ }^{21}$. Las fases del análisis fueron 2: análisis intra-casos y análisis inter-casos.

En la primera fase de análisis intra-caso los investigadores realizaron la codificación abierta, proceso en que ambos investigadores por separado codificaron parte de los libros, reconstruyendo TS explicitas o implícitas en los libros. Lo anterior implicó expresar los conceptos de esta codificación (para propósitos de este estudio TS) en una estructura de hipótesis o teoría, identificando un antecedente y un consecuente, en la mayoría de los casos utilizando la forma si... entonces $^{1}$. De esta manera se identificaron frases o párrafos textuales de cada libro que dieron origen a códigos expresados por los investigadores en forma de TS, una misma TS en ocasiones fue usada para representar a diferentes citas de un libro. También se caracterizó a cada libro con una expresión representativa de la TS presentes en él, generando lemas de caso, que es el procedimiento propuesto por Flick ${ }^{21}$ en las descripciones breves de caso que se realizan en la codificación temática. En una segunda fase los investigadores en conjunto agruparon los casos con el objetivo de realizar un análisis inter caso a partir de los resultados de la fase anterior (TS y lemas de caso). El propósito de esta codificación de los datos (codificación axial) fue la identificación de TS supraordenadas (TS que subordinan a otras) que agruparan en categorías a las TS subordinadas reconocidas en citas textuales de los libros en la primera fase y los lemas de cada libro considerando la posibilidad de que más de un libro y con diferentes autores compartieran una misma explicación general acerca de la DP.

Respecto al resguardo de los criterios de rigor científico se cauteló la transparencia; al tener el lector la posibilidad de entender cómo se llegó a los resultados. También se buscó cumplir con la credibilidad mediante triangulación del investigador en los análisis, al analizar los autores los datos de forma separada y triangulación metodológica entre métodos recurriendo para alcanzarla a diferentes procedimientos de codificación de los datos ${ }^{21}$.

Este estudio se basó en datos públicos (libros), por lo que no fue necesario someter esta investigación a un Comité de Ética.

\section{Resultados}

\section{Teorías subjetivas sobre disciplina familiar sustentadas por los libros (análisis intra-casos)}

Por motivos de espacio los resultados de esta fase se describirán mediante una tabla. Así, en tabla 2 se presentan los resultados de la fase de análisis intra-casos, para cada libro se describe: a) una afirmación considerada típica en él o lema del caso, acerca de la DP y b) la TS central de cada libro, TS que actuando como categoría central agrupó a las demás TS del libro (los códigos).

\section{Teorías subjetivas supraordenadas sobre la disciplina familiar presentes en los libros (análisis inter-casos)}

No se encontró una TS común a todos los libros analizados, sino que se reconstruyeron 3 TS supra ordenadas $^{1}$ sobre DP en las que se clasificó cada libro, 
Tabla 1. Libros Incluidos (n: 13)

\begin{tabular}{|c|c|c|c|c|c|}
\hline $\begin{array}{l}\text { Identificador } \\
\text { de casos } \\
\text { (libros) }\end{array}$ & Título & Autor & Profesión/especialidad* & $\begin{array}{l}\text { Año de } \\
\text { la edición } \\
\text { consultada }\end{array}$ & $\begin{array}{l}\text { País de la } \\
\text { edición } \\
\text { consultada }\end{array}$ \\
\hline A & $\begin{array}{l}\text { Niños con pataleta, } \\
\text { Adolescentes desafiantes }\end{array}$ & Amanda Céspedes & Psiquiatra infanto juvenil & 2017 & Chile \\
\hline B & $\begin{array}{l}\text { Hijos con personalidad... Raíces } \\
\text { y alas }\end{array}$ & $\begin{array}{l}\text { Alexander Lyford-Pike, } \\
\text { Marianella Ciompi* y } \\
\text { Mª José Soler* }^{*}\end{array}$ & Psiquiatra y psicólogas* & 2010 & Chile \\
\hline$C$ & Creciendo juntos... & Carlos González & Médico pediatra & 2013 & España \\
\hline D & $\begin{array}{l}\text { Hijo único consejos para la } \\
\text { crianza }\end{array}$ & $\begin{array}{l}\text { María Elena López y } \\
\text { María Teresa Arango }\end{array}$ & Psicólogas clínicas & 2011 & Chile \\
\hline$E$ & $\begin{array}{l}\text { A ser feliz también se aprende. } \\
\text { Guía para la educación del } \\
\text { niño de } 2 \text { a } 6 \text { años }\end{array}$ & Neva Milicic & Psicóloga educacional & 2001 & Chile \\
\hline $\mathrm{F}$ & $\begin{array}{l}\text { Hijos tiranos o débiles depen- } \\
\text { dientes }\end{array}$ & Marta Alicia Chávez & Psicoterapeuta & 2012 & México \\
\hline G & Padres que aman demasiado & $\begin{array}{l}\text { Annie de Acevedoa, } \\
\text { Jane Nelsen }{ }^{\mathrm{b}} \text { y Cheryl Erwin }\end{array}$ & $\begin{array}{l}\text { a. Psicóloga infantil } \\
\text { b. Consejera familiar y } \\
\text { de pareja } \\
\text { c. Terapeuta matrimonial y } \\
\text { familiar }\end{array}$ & 2011 & Chile \\
\hline $\mathrm{H}$ & $\begin{array}{l}\text { Los hijos en el medio. Cuidando } \\
\text { su espacio durante el divorcio }\end{array}$ & $\begin{array}{l}\text { Ana Tempelsmana y } \\
\text { Silvia Salinas }\end{array}$ & $\begin{array}{l}\text { a. Psicóloga somática } \\
\text { b. Psicóloga clínica }\end{array}$ & 2011 & Argentina \\
\hline I & Disciplina sin lágrimas & $\begin{array}{l}\text { Daniel J. Siegel* y } \\
\text { Tina Bryson** }\end{array}$ & $\begin{array}{l}\text { Médico pediatra y psiquiatra* } \\
\text { Psicoterapeuta** }\end{array}$ & 2015 & España \\
\hline J & Psicología del desarrollo & $\begin{array}{l}\text { Felipe Valdemar Olórtegui } \\
\text { Miranda }\end{array}$ & Psicólogo & $\begin{array}{l}2008\left(2^{a}\right. \\
\text { ed. })\end{array}$ & Perú \\
\hline K & $\begin{array}{l}\text { Sin dañar a terceros. El niño } \\
\text { ante los conflictos entre papá } \\
\text { y mamá }\end{array}$ & $\begin{array}{l}\text { Julia Borbolla de Niño de } \\
\text { Rivera }\end{array}$ & Psicóloga clínica & 2016 & México \\
\hline L & $\begin{array}{l}\text { No quiero crecer. Viva la dife- } \\
\text { rencia para padres con hijos } \\
\text { adolescentes }\end{array}$ & Pilar Sordo & Psicóloga & 2010 & Chile \\
\hline M & Ni rabietas ni conflictos & Rosa Jové & $\begin{array}{l}\text { Psicóloga clínica infanto y } \\
\text { juvenil y psicopediatra }\end{array}$ & 2011 & España \\
\hline
\end{tabular}

* La información sobre las profesiones y especialidades de los(as) autores(as) fue obtenida del mismo libro o de la páginas web oficiales de los autores(as).

desde nuestra interpretación basada en los resultados anteriores del análisis intra-casos y desde un polo más permisivo hasta otro más centrado en la autoridad. Estos resultados son similares a los encontrados en estudios de libros para padres de contextos diferentes al latinoamericano y de forma muy general estas TS incluyen evidencia científica sobre la DP.

La primera TS destaca la importancia de que los padres disciplinen velando por el "desarrollo emocional" y evitando prácticas basadas en la autoridad. La segunda, sugiere una mezcla de autoridad con afecto y evitación, en lo posible, del castigo. La tercera, destaca la necesidad de una disciplina firme y con "autoridad". (tabla 3).

\section{Discusión}

La primera pregunta generativa del presente trabajo es si existiría una TS base compartida sobre el concepto de disciplina familiar en los libros estudiados. En coincidencia con otra investigación previa realizada también con publicaciones de ayuda para padres escritas por autores latinoamericanos ${ }^{10}$, se encontró en esta investigación que hay una coexistencia de visiones opuestas sobre la DP posibles de expresar en diferentes TS supra ordenadas.

En general, se observa que las TS pueden ser ubicadas desde un polo que defiende la importancia de que los padres velen por el "desarrollo emocional" y la 
Tabla 2. Lemas y teorías subjetivas representativos de cada libro

\begin{tabular}{cl}
\hline $\begin{array}{c}\text { Libros } \\
\text { (casos) }\end{array}$ & Lema del caso \\
\hline A & $\begin{array}{l}\text { La rebeldía de un niño es posible abordarla, si se identifican sus } \\
\text { causas, con "límites claros constantes y flexibles", con el objetivo } \\
\text { de educarlo emocionalmente }\end{array}$ \\
B $\quad \begin{array}{l}\text { Educar con firmeza, pero en un ambiente de confianza y afecto } \\
\text { desarrolla el carácter y las virtudes humanas }\end{array}$ \\
C $\quad \begin{array}{l}\text { Se debe disciplinar con amor, respeto y paciencia, porque los } \\
\text { hijos tienen disposición a obedecer, no es necesaria la autoridad }\end{array}$
\end{tabular}
hijos tienen disposición a obedecer, no es necesaria la autoridad

D La culpa de los padres por tener un solo hijo a veces es proyectada a los hijos, cediendo ante los deseos del hijo, esto genera: hijos manipuladores y exigentes

E Si las normas y la disciplina se han establecido bien, no es necesario imponer autoridad

F La falta de autoridad genera hijos sobreprotegidos, gracias a padres débiles que temen incomodarlos

H Los límites son necesarios, pero deben establecerse con cariño y presencia.

I "Disciplina con empatía y amor para el desarrollo integral del hijo"

J El "castigo" (o "sanciones") físico y psicológico, origina heridas que no sanan

K "Las normas claras definen espacios claros. Dentro de ellos es más fácil jugar y aprender a vivir" (p. 135)

L " "La autoridad es necesaria, porque genera limpieza, coherencia y la sensación de estar en un mundo seguro" (p. 28)

M Los límites y los castigos son innecesarios si los padres saben "conseguir con otras técnicas" "educar o cambiar una conducta"
Teoría Subjetiva

Es necesario "ir a la causa" de un comportamiento rebelde para comprender su origen y así poder abordarlo

Si la DP es ejercida con firmeza, con hechos y autoridad, entonces se logra educar hijos maduros y felices

Las características de la sociedad actual (diversidad cultural) genera en los padres dudas acerca de la DP, lo que repercute en ellos con sentimientos de culpa, inseguridad y estrés, y conlleva a implementar un estilo disciplinario principalmente autoritario repercutiendo en dificultades en su desarrollo. Por lo tanto, para remediarlo, los padres deben aprender a ser indulgentes, enseñar desde el diálogo y la tolerancia

Si se educa con permisividad, entonces el niño no aprende a ser responsable y con autocontrol

Si los niños comprenden las consecuencias de sus conductas y lo que se espera de ellos, entonces no es necesario castigarlos

Si hay disciplina y no sobreprotección, entonces es posible criar hijos autónomos y responsables y evitar conflictos familiares

Si los "límites", castigos, normas o reglas están acompañados de "cariño", entonces ahí cumplen su objetivo; estar al servicio del "cuidado" y del crecimiento personal de los hijos (se ponen por amor a ellos)

El niño muestra indisciplina por su inmadurez cerebral, la tensión que le afecta, la circunstancia o el apego seguro que mantiene con su progenitor. Así, para disciplinar debemos controlarnos emocionalmente, empatizar, expresar amor y desde allí promover la colaboración inmediata y el desarrollo cerebral del hijo

Si los padres gritan al niño esto los marca y genera trastornos de personalidad o comportamientos violentos

Los límites dan seguridad a los hijos, eso permite que sepan qué pueden hacer y qué no, lo que genera que aprendan a hacerse responsables de sus acciones y sus consecuencias

Si no aplicamos "autoridad", "límites" y orden, entonces no se le está dando al niño lo que necesita

Si hacemos que los hijos obedezcan a través de castigos (y sin ellos entender el motivo), entonces estamos criando futuros adultos sumisos, ahí la educación de ellos habría fracasado

TS: teoría subjetiva; DP: Disciplina parental. " ": cuando el concepto o frase fue tomado textualmente del libro.

evitación de prácticas autoritarias, hasta otro en donde la disciplina firme y con autoridad se ve como facilitadora de la educación familiar. Lo anterior pareciera tener de base dos visiones contrapuestas del niño(a) y sus necesidades: (a) un polo respetuoso que concibe al niño(a) como una persona con derechos y libertades que no deben ser coartadas; (b) otro polo en donde pareciera verse al niño(a) como un futuro adulto, que desde sus primeros años debe aprender virtudes y valores como el respeto a las normas y la buena adaptación social, extremo que defiende la importancia de la firmeza en la crianza. Los resultados sobre las TS de los libros muestran en general correspondencia con los estilos de crianza que han sido estudiados en diferentes contextos, representándose aquí creencias cercanas al estilo permisivo o democrático y otras al autoritativo ${ }^{12}$. 
Tabla 3. Características de tres teorías subjetivas generales acerca de la disciplina parental y los libros que las representan

Teorías Subjetivas Generales (supra ordenadas)

1

La (DP) basada en el amor, respeto y empatía, no requiere de autoridad ni de castigos

La DP debe tener firmeza y afecto; la firmeza puede requerir algunos límites y castigos y el afecto es muy importante, porque entre ambos se logra un fin educativo

Características de cada TS

1. Estilo (disciplinario) comprensivo, amoroso, respetuoso y empático, en donde el concepto de autoridad no es fundamental. Rechazo de los castigos y se propone el uso del diálogo para que los hijos comprendan las consecuencias de sus actos mediante la reflexión. Se utilizan menos límites y se otorga más libertad. Este estilo facilitaría la comunicación y conlleva al desarrollo de hijos saludables y maduros

Libros que sustentan cada TS

C, I, M
2. Los límites (no agobiantes) y las sanciones son necesarios. La DP debe ser una mezcla de "autoridad", afecto y cariño. Los padres deben poner algunos límites y "normas" a sus hijos y solo si es necesario, utilizar algunos castigos o técnicas como el "tiempo fuera". Se valora que los padres sean "firmes", pero flexibles
La educación adecuada de los hijos, requiere de límites, control, autoridad, en ocasiones, de castigos y de padres que no sientan culpa. Sin lo anterior se pierde el respeto y/o se manifiesta la sobreprotección

3. Estilo "Firme" (valor del respeto, reglas, sí "autoridad", sí castigo con cariño - incluido el físico para algunos-). DP positiva, no excesos, pero sí control y límites. Culpa de los padres como causa de la sobreprotección. Educar como propósito de la DP

A, B, D, F, G, K, L, H

1. TS: teoría subjetiva; 2. DP: Disciplina parental. 3. " ": cuando el concepto o frase fue tomado textualmente de alguno de los libros que pertenecen a una de las 3 TS.

El rol del castigo es un elemento claramente diferenciador entre las tres TS, desde la no recomendación de los castigos, el privilegio de la contención emocional y de la explicación moral hacia los(as) hijos(as) $(\mathrm{TS} 1)^{6}$, pasando en lo posible por la evitación del castigo (TS2), hasta una TS general en donde las técnicas disciplinarias incluyen el castigo, siguiendo principios de origen conductista como el dejar llorar, lo que se describe como la manipulación por parte del hijo(a) (TS3). Lo anterior coincide con el estudio de las diferentes técnicas disciplinarias preferidas por los padres, las que sabemos dependen de factores como su escolaridad, el género ${ }^{13}$ o la edad ${ }^{22}$. Los libros estudiados coinciden, salvo una excepción, en la no recomendación de todo tipo de castigo físico, estando en línea con las investigaciones actuales ${ }^{23}$, que muestran consenso de que incluso el castigo físico leve (palmadas, nalgadas) se asocia a diferentes problemas en la vida futura de los niños ${ }^{19}$. Este resultado coincide con el hallazgo de Cain ${ }^{8}$, quien encontró que las publicaciones que apoyan el castigo físico leve son una minoría.

En términos más amplios, este resultado podría apoyar la hipótesis de Gergen ${ }^{24}$ sobre la presencia simultánea en la sociedad de un ideal de sujeto propio de la modernidad (polo de la firmeza para la inserción adecuada en la sociedad), junto a una visión del sujeto como un ser libre, en donde lo importante es su desarrollo integral (individual), en un contexto cultural con tendencia al "desgaste de la autoridad" ("con- ciencia posmoderna"), desarrollo de los niños(as) que se vería afectado de manera importante a causa de la crianza ejercida por los padres ${ }^{11}$. Por último, es posible hipotetizar que estas creencias opuestas también se relacionan, en uno de sus extremos, con una visión menos conservadora de la educación de los hijos y con una tendencia al niño-centrismo en la crianza (TS1) ${ }^{25}$, opuesta a una perspectiva centrada en el desarrollo y mantención de la familia como institución, en donde la indisciplina o una "crisis de autoridad" podría acarrear conflictos que la debiliten.

Sobre la tendencia hacia "la firmeza" en la crianza de los(as) hijos(as) para evitar la "mala disciplina", es interesante señalar que hay registros en Argentina de su divulgación a través de publicaciones para padres, escritas por profesionales de la salud en la década de los treinta ${ }^{11}$. Lo anterior muestra cómo este enfoque de asesoramiento hacia los padres es de larga data en Latinoamérica y según los resultados de este estudio, está aún presente.

En segundo lugar, nos preguntamos en este trabajo si se observan en los libros analizados tendencias similares a otras revisiones de este tipo de libros en contextos diferentes al latinoamericano. No encontramos cambios en las posiciones predominantes por época a través de la historia sobre la crianza de los(as) hijos(as), como lo descrito por Hardyment ${ }^{7}$. En nuestro estudio aparece el desacuerdo respecto de la DP, con argumentos que hacen poca referencia al conocimiento cientí- 
fico generado por las disciplinas a las que pertenecen los(as) autores(as), pero sí al conocimiento profesional e implícitamente asociado a valores, coincidente con otros estudios realizados en contextos diferentes?.

Finalmente, la tercera pregunta generativa de este trabajo es si se observaría en las TS la incorporación de la investigación en DP. Se encontró que solo de forma muy general están implícitos o explícitos estudios empíricos en algunas de las TS. Lo que caracteriza a estos libros es la argumentación fundamentada en la experiencia profesional e implícitamente en algunas corrientes psicológicas. En su mayoría no se observa una divulgación del conocimiento científico contemporáneo relativo a la disciplina parental, por ejemplo, efectos de las diferentes técnicas disciplinarias, su eficacia, los factores contextuales asociados o que actúan como mediadores entre su uso y sus efectos en el desarrollo de los niños(as) y adolescentes ${ }^{26}$. Tampoco consideraciones contextuales relativas al nivel socioeconómico o diferencias según el género de los hijos, que los estudios científicos muestran ${ }^{15}$. Según la evidencia científica, se ha demostrado en forma importante que los extremos de la disciplina, ya sean excesivamente permisiva o excesivamente punitiva, conducen a consecuencias perjudiciales para los niños(as) ${ }^{12}$.

Investigaciones sobre TS o creencias, realizados con profesionales (médicos, enfermeras, psicólogos y educadores entre otros) muestran resultados similares a los del presente estudio, esto es, que en los profesionales coexisten ${ }^{27}$ (a veces predominan) teorías subjetivas, basadas en su experiencia individual (personal o profesional $)^{2}$, junto a teorías científicas ${ }^{28,29}$ o basadas en aprendizajes provenientes de la formación, al tomar decisiones o describir algunos aspectos de su trabajo ${ }^{30}$. Como afirma Durán ${ }^{31}$, destacando la idea de Schön ${ }^{32}$, en el nivel aplicado del trabajo profesional, los problemas se resisten a soluciones técnicas o basadas en la teoría ${ }^{31}$. Es posible hipotetizar que, en aquellos dominios más cercanos a la vida personal del profesional, en este caso cómo fue criado o cómo cría, éstas TS tengan mayor fuerza que la evidencia científica que fundamenta su título o especialidad.

Por último, queremos extender estos resultados con cautela al acto de asesoramiento a los padres dados por profesionales. De observarse la misma tendencia presente en este estudio, podríamos suponer efectos de confusión o incluso de posibles enjuiciamientos de profesionales a padres, basados en preferencias personales respecto a prácticas disciplinarias. Sobre esta hipótesis, hay estudios sobre los efectos no deseados del asesoramiento profesional a padres sobre crianza ${ }^{5}$.

Este estudio tiene limitaciones: la ausencia de más procedimientos de triangulación en el análisis, incorporar otras técnicas de análisis de TS pudiera ser un aporte y un muestreo realizado desde diferentes países hispanohablantes permitiría mejorar la reconstrucción del objeto de estudio.

Una fortaleza de esta investigación es ser la primera en Latinoamérica y España que analiza este tipo de libros, provenientes de diferentes países y focalizándose en aquellos escritos por profesionales de la salud y en el tema de la DP.

Los resultados de este trabajo alertan de la necesidad de continuar estudiando los consejos profesionales sobre disciplina, para comprender mejor la naturaleza de este tipo de conocimiento, su impacto en el desarrollo del niño(a) y en el rol parental. Es importante que las asociaciones profesionales de Hispanoamérica y España trabajen en elaborar consensos basados en evidencia sobre este tema y sobre otros relacionados a la crianza.

\section{Conclusiones}

En conclusión, se han identificado 3 teorías subjetivas supraordenadas ${ }^{1}$, con las que es posible diferenciar los libros para padres con consejos sobre DP revisados en este estudio. En relación a la información utilizada en los libros revisados, los resultados evidencian que solo de manera muy general se utiliza implícita o explícitamente la evidencia científica actual sobre el tema. Por último, los libros contienen consejos fundamentados principalmente en TS basadas en la experiencia profesional y en visiones particulares de la sociedad y del rol de la familia, e implícitamente y de manera no preponderante en el conocimiento ligado a algunas corrientes médicas o psicológicas.

Lograr estandarizar las recomendaciones para padres y/o cuidadores sobre DP entregadas por el equipo de salud y elaborar guías prácticas al respecto, podría contribuir a generar vínculos y relaciones sanas entre padres e hijos. Lo anterior para ayudar a los niños(as) a alcanzar un desarrollo intelectual y emocional saludable mediante el uso de prácticas disciplinarias respetuosas que además se extienda a las siguientes generaciones.

\section{Responsabilidades éticas}

Protección de personas y animales: Los autores declaran que los procedimientos seguidos se conformaron a las normas éticas del comité de experimentación humana responsable y de acuerdo con la Asociación Médica Mundial y la Declaración de Helsinki.

Confidencialidad de los datos: Los autores declaran que han seguido los protocolos de su centro de trabajo sobre la publicación de datos de pacientes. 
Derecho a la Privacidad y Consentimiento Informado: Los autores declaran que la información ha sido obtenida de datos previos en forma anonimizada, por lo cual el Comité de Ética de Investigación en uso de sus facultades, ha eximido de la obtención de un con- sentimiento informado, lo cual consta en el acta respectiva.

\section{Conflicto de intereses}

Los autores declaran no tener conflicto de intereses.

\section{Referencias}

1. Catalán J. Teorías subjetivas. Aspectos teóricos y prácticos. La Serena: Editorial Universidad de La Serena;2010.

2. Hatzidimitriadou E, Psoinos M. Cultural health capital and professional experiences of overseas doctors and nurses in the UK. Divers Equal Health Care. 2014;11(1):35-47.

3. Kada O, Hedenik M, Griesser A, Mark AT, Trost J. Subjektive theorien zu lebensqualität und gesundheit im alter: eine explorative studie mit pflegeheimbewohnern und ihren pflegekräften. Z Gerontol Geriatr. 2018:628-35.

4. Kraus A. Scholarly principles in teacher education: What kind of science serves a practice-oriented teacher education? European Research on Educational Practices. Münster. Waxmann Verlag; 2015.

5. Davis A. Experts and childcare 'bibles': mothers and advice literature. En: Davis A, Modern Motherhood: Women and Family in England, 1945-2000, 1a Ed. Manchester. Manchester University Press 2012:112-41.

6. Larzelere R, Kuhn B. Parental discipline. En: Shehan CL, The Wiley Blackwell Encyclopedia of Family Studies, $1^{\text {a }}$ Ed. New Jersey: Wiley Online Library 2016;19; doi: 10.1002/9781119085621.wbefs512/ full.

7. Hardyment C. Dream babies: childcare advice from John Locke to Gina Ford. 1st rev. Londres: Frances Lincoln; 2007.

8. Cain DS. Parenting online and lay literature on infant spanking: information readily available to parents. Soc Work Health Care. 2008;47(2):174-184.

9. Ramos KD, Youngclarke, DM. Parenting advice books about child sleep: cosleeping and crying it out. Sleep. 2007;29(12):1616-23.

10. Fuentes-Vega S, Castro PJ, Van der Veer, R. Cosleeping versus sleep training: parenting advice publications. Rev Fac Med. 2017;65(2):203-10.
11. Borinsky M. "Todo reside en saber qué es un niño". Aportes para una historia de la divulgación de las prácticas de crianza en la Argentina. Anu Investig Fac Psicol Univ B Aires. 2005;13:117-26.

12. Baumrind D, Larzelere R, Owens E. Effects of preschool parents' power assertive patterns and practices on adolescent development. Parent-Sci Pract. 2010;10(3):157-201.

13. Hallers-Haalboom ET, Groeneveld MG, van Berkel SR, et al. Wait until your mother gets home! Mothers' and fathers' discipline strategies. Soc Dev. 2016;25(1):82-98.

14. Pereira M, Negrão M, Soares I, Mesman J. Decreasing harsh discipline in mothers at risk for maltreatment: a randomized control trial. Inf Mental Hlth J. 2014;35(6):604-13.

15. Aguirre E, Villavicencio C. Parenting styles in adolescents with aggressive behavior. Conference Proceedings 2017;1:68-71; http://investigacion. utmachala.edu.ec/proceedings/index.php/ utmach, última visita 7-10-2017.

16. Gershoff ET, Grogan-Kaylor A. Spanking and child outcomes: Old controversies and new meta-analyses. J Fam Psychol. 2016;30(4):453-69.

17. Sáenz-Lozada ML, Camacho-Lindo AE, Silva-Oviedo NJ, Holguin-Sanabria A. Exploración de estrategias de disciplina aplicadas a los niños de una institución educativa en Bogotá. Rev Fac Med. 2014;62(2):199-204.

18. Strauss A. (1987). Qualitative analysis for social scientists. Cambridge: Cambridge University.

19. Castro PJ, van der Veer R, BurgosTroncoso G, Meneses-Pizarro L, Pumarino-Cuevas N, TelloViorklumds C. Teorías subjetivas en libros latinoamericanos de crianza, acerca de la educación emocional. Rev Latinoam Cienc Soc Niñez Juv. 2013;11(2):703-18

20. Glaser BG, Strauss A. The discovery of grounded theory, Chicago: Aldine Publishing Company, 1967.
21. Flick U. An introduction to qualitative research. 4th ed., London: Sage Publications, Inc., 2010.

22. Smith ML, Chiappone AL, Wilson KL. Parenting and child rearing attitudes of unmarried Hispanic teenage mothers. J Child Fam Stud. 2017;26:643.

23. Gershoff ET, Grogan-Kaylor A. Spanking and child outcomes: old controversies and new meta-analyses. J Fam Psychol. 2016;30(4):453-69.

24. Gergen K. El Yo Saturado. Dilemas de identidad en el mundo contemporáneo. Paidós, Barcelona, 1997.

25. Ashton-James CE, Kushlev K, Dunn EW. Parents reap what they sow: child-centrism and parental well-being. Soc Psychol Personal Sci [Internet]. 2013 Mar 14;4(6):635-42; doi: $10.1177 / 1948550613479804$.

26. Germán M, Gonzales N, McClain DB, Dumka L, Millsap R. Maternal warmth moderates the link between harsh discipline and later externalizing behaviors for Mexican American adolescents. Parent-Sci Pract. 2013;13(3):169-177.

27. Najavits L. Psychotherapists' implicit theories of psychotherapy. J Psychother Integr. 1997;7:1-16.

28. Grol R. Beliefs and evidence in changing clinical practice. BMJ. 1997;315:418-21.

29. Castro-Carrasco PJ, Alaniz PB, Carmona DM, Pizarro TP, Soto CM, Fuster DT. What do Chilean and Costa Rican psychologists believe and advise about parenting? Act. Psic. 2017;31(123):43-60.

30. Cuadra-Martínez D, Castro PJ, Vystr ilová $\mathrm{P}$, Jancic Mogliacci R. A review of research on teachers' subjective theories: contributions to the study of teacher education. Psychol Educ. 2017;54(3):1-22.

31. Durán P. Nutrición temprana y enfermedades en la edad adulta: acerca de la "hipótesis de Barker". Arch argent pediatr. 2004;102(1):26-34.

32. Schön D. La Formación de Profesionales Reflexivos. Hacia un nuevo diseño de la enseñanza y el aprendizaje en las profesiones. Paidós, Barcelona, 1992. 